\title{
Análisis biomecánico de la marcha en paciente con secuelas de poliomielitis y artroplastia de rodilla
}

\section{Clinical gait analysis in patient with poliomyelitis sequelae and total knee arthroplasty}

\section{Análise biomecânica da marcha em um paciente com sequelas de poliomielite e artroplastia do joelho}

\author{
David López, ORCID 0000-0001-9748-6964 \\ Nicolás Peña, ORCID 0000-0002-3654-7636 \\ Ernesto Benítez, ORCID 0000-0002-1858-2948 \\ Sofía Samudio, ORCID 0000-0001-7399-9597 \\ Pilar Vecino, ORCID 0000-0002-4125-2947 \\ Eugenia Benvenutto, ORCID 0000-0002-9025-6987
}

Universidad Católica del Uruguay

Recibido: 23/12/2020

Aceptado: 17/05/2021

\begin{abstract}
Resumen: La poliomielitis es una enfermedad que puede provocar secuelas irreversibles, generando pérdida de fuerza muscular, parálisis e hiporreflexia, entre otras. Hoy en día las infecciones por poliovirus están controladas, pero se siguen tratando personas con secuelas que pueden ver afectada su calidad de vida y funciones cotidianas, tales como la marcha. Habiendo agotado las opciones de tratamiento conservadoras, la artroplastia total de rodilla (ATR) es una de las intervenciones quirúrgicas más habituales cuando las secuelas afectan la morfología y funcionalidad de dicha articulación. El objetivo del estudio es realizar un análisis instrumentado de la marcha de un paciente con secuelas de poliomielitis y una ATR, con el fin de definir las mejores estrategias de rehabilitación y mejorar la recuperación de la máxima funcionalidad. El estudio se realizó en un Laboratorio de Análisis de Movimiento con 8 cámaras, mediante la colocación de marcadores reflectivos en el cuerpo del paciente. Los resultados muestran alteraciones del patrón de marcha en todas las articulaciones de las extremidades inferiores y en cada uno de los planos anatómicos, siendo la más relevante la rotación interna de la articulación de la cadera derecha y una flexión fija en $9^{\circ}$ de la articulación de la rodilla ipsilateral, durante la primera mitad del ciclo de marcha. El análisis sugiere que el paciente adopta estrategias que favorecen la activación del tensor de la fascia lata como flexor de cadera y estabilizador de la articulación de la rodilla en la máxima extensión disponible (9 ○ de flexión), en sustitución del músculo cuádriceps, debilitado debido a las secuelas de la poliomielitis.
\end{abstract}

Palabras clave: síndrome pospoliomielitis; artroplastia de reemplazo de rodilla; análisis de marcha; fenómenos biomecánicos. 


\begin{abstract}
Poliomyelitis is a disease that may cause irreversible sequelae, generating muscle strength deficits, flaccid paralysis and hyporeflexia, among others. Even though poliovirus infections are under control, people with sequelae that can affect their quality of life and everyday functions such as walking, are still being treated. When these sequelae affect the morphology and functionality of the knee joint, and every other conservative option has been exhausted, the total knee arthroplasty (TKA) is one of the most common surgical interventions. The main goal of the present study is to perform an instrumented gait analysis test of a patient with poliomyelitis sequelae and TKA, in order to define the best rehabilitation strategy and achieve the highest level possible of the patient's function. 3D Gait Analysis was performed with an 8-camera motion capture system and reflective markers placed on specific body landmarks. Results show gait pattern disturbances in every joint and at every anatomical plane, being the most relevant the right hip internal rotation and a fixed 9 degrees ipsilateral knee flexion during the first half of the gait cycle. The analysis suggests that the patient adopts strategies that promote the activation of the tensor fascia lata as hip flexor and knee stabilizer at the maximum available extension $\left(9^{\circ}\right.$ of flexion) replacing the weakened quadriceps muscle due to the poliomyelitis sequelae.
\end{abstract}

Keywords: post-poliomyelitis syndrome; arthroplasty replacement knee; gait analysis; biomechanical phenomena.

Resumo: A poliomielite é uma doença que pode causar sequelas irreversíveis, causando perda de força muscular, paralisia e hiporreflexia, entre outras. Hoje as infecções por poliovírus são controladas, mas ainda existen pessoas en tratamento pelas sequelas que podem danificar sua qualidade de vida e funções diárias, como caminhar. Esgotadas as opções de tratamento conservador, a artroplastia total do joelho (ATJ) é uma das intervenções cirúrgicas mais comuns quando as sequelas afetam a morfologia e a funcionalidade dessa articulação. $\mathrm{O}$ objetivo deste trabalho é realizar uma análise instrumentada da marcha de um paciente com sequela de poliomielite e ATJ, a fim de definir as melhores estratégias de reabilitação e melhorar a recuperação da funcionalidade máxima. O estudo foi realizado em um Laboratório de Análise de Movimento com 8 câmeras, por meio da colocação de marcadores reflexivos no corpo do paciente. Os resultados mostram alterações no padrão de marcha em todas as articulações dos membros inferiores e em cada um dos planos anatômicos, sendo as mais relevantes a rotação interna do quadril direito e a flexão fixa do joelho ipsilateral a $9{ }^{\circ}$ durante o primeiro ciclo do meio da marcha. A análise sugere que o paciente adote estratégias que favoreçam a ativação do tensor da fáscia lata como flexor do quadril e estabilizador do joelho na extensão máxima disponível $\left(9{ }^{\circ}\right.$ de flexão), substituindo o músculo quadríceps, enfraquecido pelas sequelas de poliomielite.

Palavras-chave: síndrome pós-poliomielite; artroplastia do joelho; análise de marcha; fenômenos biomecânicos.

Correspondencia: Ernesto Benítez, Departamento de Ciencias del Movimiento Humano, Universidad Católica del Uruguay.E-mail: ernesto.benitez@ucu.edu.uy 


\section{Introducción}

La poliomielitis es una enfermedad altamente infecciosa provocada por el poliovirus que predominantemente afecta a niños menores de 5 años y que provoca secuelas duraderas en la edad adulta (1). Se caracteriza por una pérdida de neuronas motoras, que pueden causar parálisis flácida, pérdida muscular e hiporreflexia (1-2). Aunque afecta principalmente a uno o ambos miembros inferiores (MMII) con severidad y extensión variable, puede afectar también a cualquier unidad motora con predominio de la cintura escapular y la espalda baja (3). Los casos afectados por poliovirus bajaron considerablemente a nivel mundial, desde 350.000 casos en 1988 a tan solo 35 casos registrados en el 2018 (4).

Dado que los pacientes con parálisis flácida de la extremidad inferior desarrollan hipotonía generalizada, alineaciones anormales y laxitud, y sumado al hecho de la alta frecuencia de afectación del músculo cuádriceps (indispensable durante ciertas fases de la marcha), estos pacientes presentan predisposición a sufrir patologías en la articulación de la rodilla a edades tempranas (2). A pesar de la existencia de estrategias terapéuticas para intentar resguardar la articulación de forma temprana, que mejoran la alineación y aumentan la estabilidad, una vez instaurada una osteoartritis dolorosa sumada a la laxitud ligamentosa (que ocasionalmente desencadena una hiperextensión dolorosa de la articulación de la rodilla), la artroplastia total de rodilla (ATR) estaría indicada en estos pacientes (1-2).

La ATR en estos pacientes supone un reto técnico por las características anatómicas aberrantes de todos los tejidos que conforman esta articulación (2). Estas alteraciones incluyen deformidades articulares y metafisarias, aumentada rotación tibial externa, alineaciones en valgo excesivo, pérdida de hueso, estrechamiento del canal femoral y tibial, genu-recurvatum y laxitud ligamentosa (2). Adicionalmente, los perfiles rotacionales de las extremidades inferiores de estos pacientes predisponen en muchos casos a patelas subluxadas o dislocadas de forma crónica (1-2). A pesar de lo anteriormente mencionado, los hallazgos apoyan el uso de ATR en los pacientes con rodillas afectadas por secuelas de poliomielitis (5). Los resultados funcionales postoperatorios son similares a otros tipos de pacientes con ATR, sin embargo, las tasas de revisión de los implantes son mayores en los pacientes post poliomielíticos (1-2, 5-7).

La fuerza muscular del cuádriceps parece ser un factor pronóstico importante para los resultados funcionales y cuando esta es menor que la antigravitatoria, el uso de implantes constreñidos está recomendado (8). Además, el uso de implantes abisagrados está indicado en pacientes más severamente afectados con pobre fuerza muscular que no proporciona estabilidad intrínseca, aumentando el riesgo de recurrencia de hiperextensión de rodilla y laxitud ligamentosa (9). Los pobres resultados a largo plazo del uso de ATR convencionales también apoyan el uso de implantes constreñidos $(7,10,11)$. Tigani et al. no justifican el criticismo previo con respecto al uso de implantes constreñidos, ya que los diseños abisagrados rotatorios modernos replican más fielmente la cinemática fisiológica articular, reduciendo además el estrés en la interfaz del implante con el hueso (7).

En la cinemática de marcha normal, el período donde el pie se encuentra en contacto con el suelo se denomina apoyo, representa el $60 \%$ del ciclo de marcha y está dividido en 5 fases: contacto inicial, respuesta a la carga, apoyo medio, apoyo terminal y prebalanceo. Cada fase tiene distinta duración y responde a una demanda funcional particular (3). 
Las primeras dos fases (contacto inicial y respuesta a la carga) componen la tarea denominada aceptación del peso, una desafiante actividad durante la marcha que implica la absorción del impacto, la estabilidad inicial en la descarga de peso y la preservación de la progresión. Durante la respuesta a la carga ocurre una rápida flexión de la articulación de la rodilla de unos $20^{\circ}$ aproximadamente, que es el principal mecanismo de absorción del impacto. El cuádriceps se activa de manera excéntrica para controlar este movimiento, generando un momento de fuerza extensor y desacelerando la velocidad de la flexión de la rodilla (3).

La aceptación del peso es seguida del soporte monopodal, otra desafiante tarea que implica que el miembro en estudio es responsable de soportar toda la carga corporal en tanto la progresión de la marcha continúa. Esta tarea se divide en las fases de apoyo medio y terminal, diferenciados principalmente por sus mecanismos de progresión. Al iniciar el apoyo medio la articulación de la rodilla comienza a extenderse debido en gran medida a la cupla flexora plantar-extensora de rodilla, un mecanismo que corresponde a la acción excéntrica de los flexores plantares (principalmente el sóleo) durante la primera parte del apoyo medio, que controla la progresión del vector de la reacción del suelo sobre el pie en apoyo. Esto resiste el momento flexor externo generado por el vector que pasa por detrás de la articulación de la rodilla, creando una aceleración en sentido opuesto, que asiste en su correcta extensión.

Dada la importancia de la deambulación en el ser humano y debido a las alteraciones de los patrones de marcha que se producen en los pacientes con secuelas de poliomielitis, los autores del estudio consideran oportuno realizar una caracterización de la cinemática de la marcha en un paciente de estas características. A través del análisis de la información que facilitan estos estudios, es posible generar estrategias en el campo de la rehabilitación de estos pacientes y mejorar la recuperación de la máxima funcionalidad en las actividades de la vida cotidiana (12-13).

\section{Presentación del caso}

Paciente de sexo femenino, de 76 años con secuelas de poliomielitis a predominancia de MMII, que cursó la patología a los 5 meses de vida. En el año 2017 se le realizó una ATR derecha, ya que la calidad de vida estaba afectada por dolor y desmejoramiento en actividades de la vida diaria. En el año 2018 se realiza una evaluación en el Laboratorio de Análisis de Marcha y Movimiento Universitario. El laboratorio cuenta con un sistema de 8 cámaras de captura de movimiento de 2.2 megapíxeles (Vicon Motion System, Oxford, UK). Se realizó un examen físico donde se valoró la fuerza muscular y el rango articular de MMII utilizando pruebas de fuerza manuales (14) y goniometría, respectivamente (15; Tabla 1). 
Tabla 1 - Valoración articular y muscular

\begin{tabular}{|c|c|c|c|c|c|c|c|c|}
\hline \multirow[t]{2}{*}{ Fuerza muscular } & & & Rango articular & & & Test de acortamiento muscular & MI Der & MI Izq \\
\hline & Grado (0-5) & Grado (0-5) & & Grados $\left({ }^{\circ}\right)$ & Grados $\left({ }^{\circ}\right)$ & Test de Ober & Positivo & Negativo \\
\hline Cadera & MI Der & MI Izq & Cadera & MI Der & MI Izq & Test de Ober modif. & Positivo & Negativo \\
\hline Flexión & 4 & 4 & Flexión & 100 & 110 & Test de Thomas & Negativo & Negativo \\
\hline Extensión & 4 & 4 & Extensión & 20 & 15 & Isquiosurales & Positivo & Positivo \\
\hline Aducción & 4 & 4 & Aducción & 30 & 29 & Cuadrado lumbar & Negativo & Positivo \\
\hline Abducción & 4 & 4 & Abducción & 36 & 31 & Test de Ely & Positivo & Positivo \\
\hline Rot int & 4 & $\begin{array}{l}4 \\
3\end{array}$ & Rot int & 40 & 40 & & & \\
\hline Rot ext & 4 & & Rot ext & 45 & 37 & Long. De MMII & $84,5 \mathrm{~cm}$ & $84,5 \mathrm{~cm}$ \\
\hline \multicolumn{9}{|l|}{ Rodilla } \\
\hline Flexión & 4 & 4 & Rodilla & & & & & \\
\hline \multirow[t]{2}{*}{ Extensión } & 2 & 3 & Flexión & 110 & 122 & & & \\
\hline & & & Extensión & -9 & 0 & & & \\
\hline Tobillo & & & Torsión tibial & 43 & 31 & & & \\
\hline Dorsiflexión & 4 & 4 & & & & & & \\
\hline Flexión plantar & 3 & 2 & Tobillo & & & & & \\
\hline Inversión & 4 & 4 & Dorsiflexión & 15 & 20 & & & \\
\hline Eversión & 3 & 0 & Flexión plantar & 48 & 35 & & & \\
\hline \multicolumn{9}{|l|}{ Raquis } \\
\hline Extensores & & 3 & unilateral & 35 & 35 & & & \\
\hline Flexores & & 3 & $\begin{array}{l}\text { Ángulo poplíteo } \\
\text { bilateral }\end{array}$ & 30 & 30 & & & \\
\hline
\end{tabular}

Se colocaron marcadores reflectivos en pelvis, muslos, piernas y pies (Figura 1a), para que sean captados por las cámaras de captura de movimiento y poder realizar un estudio instrumentado de la marcha. A nivel de pelvis, los marcadores se ubican en las espinas ilíacas anterosuperiores y posterosuperiores. En el muslo se coloca un marcador en el cóndilo femoral externo y 3 marcadores auxiliares (cluster) no anatómicos aproximadamente en el tercio distal. En la pierna se coloca un marcador en la tuberosidad anterior de la tibia, y al igual que en muslo, 3 marcadores auxiliares no anatómicos aproximadamente en el tercio distal. En los pies se coloca un marcador en la cabeza del segundo metatarsiano y en el calcáneo. Se colocan además marcadores auxiliares en las rótulas, y se determinan marcadores virtuales para cóndilo femoral interno, maléolos externo e interno. Este conjunto de marcadores permite calcular dos modelos biomecánicos simultáneamente. El más utilizado es el Modelo Convencional de Marcha, que agrupa varios modelos similares entre sí, pero con pequeñas variaciones (16-18). A pesar de ser el más conocido, este modelo tiene algunas limitaciones que podrían comprometer la calidad de los datos (19). Otros modelos biomecánicos utilizan una mayor cantidad de marcadores para compensar las limitaciones del modelo convencional (20-21). Estos modelos permiten identificar y seguir distintos segmentos del cuerpo de manera independiente (Figura 1b) y así realizar una mejor estimación de los centros y ejes articulares de manera dinámica mediante una calibración funcional (22-25). 
Figura 1 - a) Set de marcadores reflectivos para análisis instrumentado de la marcha b) Marcadores, ejes y sistemas de referencia
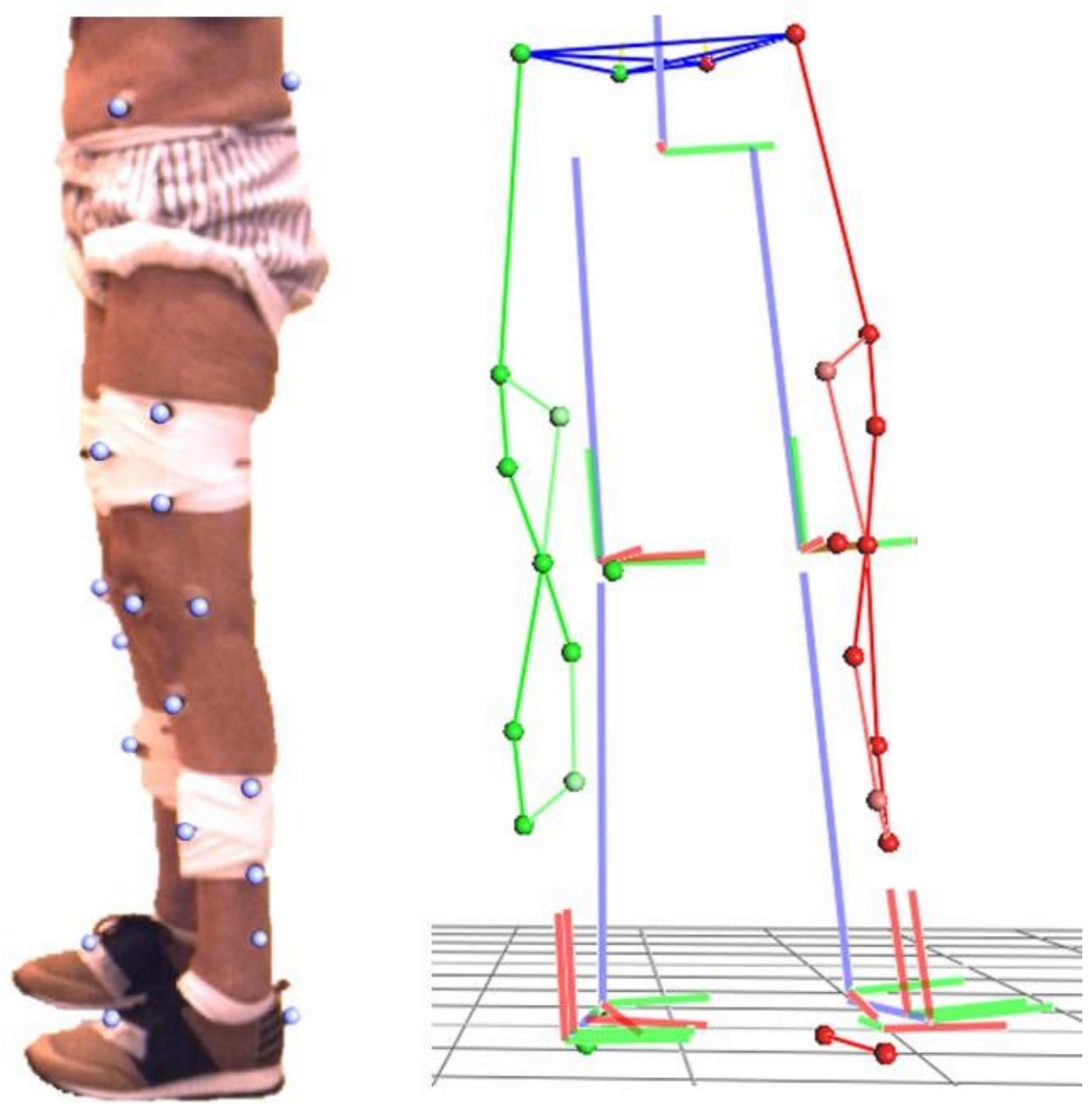

\section{Resultados}

Los resultados del análisis cinemático de la marcha muestran un patrón consistente, con un aumento del tiempo de apoyo bilateral. En relación con los parámetros espaciotemporales (Tabla 2), se puede ver una disminución del largo del ciclo de marcha, largo del paso, cadencia y la velocidad, siendo esta última aproximadamente la mitad de la normal. En relación con la longitud del paso, el paciente presenta una asimetría, siendo el derecho un $20 \%$ más corto que el izquierdo (Tabla 2). 
Tabla 2 - Parámetros espaciotemporales de la marcha

\begin{tabular}{|c|c|c|c|c|c|c|c|c|}
\hline \multirow{2}{*}{ Parámetro } & \multirow{2}{*}{ Unidad } & \multirow{2}{*}{$\begin{array}{l}\text { Grupo de } \\
\text { control }\end{array}$} & \multicolumn{2}{|c|}{ Trial 1} & \multicolumn{2}{|c|}{ Trial 2} & \multicolumn{2}{|c|}{ Trial 3} \\
\hline & & & izq & der & izq & der & izq & der \\
\hline Cadencia & pasos/min & 122.0 & 81.6 & 81.1 & 75.9 & 81.1 & 77.4 & 75.5 \\
\hline Velocidad & $\mathrm{m} / \mathrm{s}$ & 1.2 & 0.6 & 0.6 & 0.6 & 0.6 & 0.6 & 0.6 \\
\hline Tiempo de ciclo & $\mathrm{s}$ & 1.0 & 1.5 & 1.5 & 1.6 & 1.5 & 1.6 & 1.6 \\
\hline Tiempo de paso & s & 0.5 & 0.7 & 0.7 & 0.8 & 0.7 & 0.8 & 0.8 \\
\hline Despegue contralateral & $\%$ & 9.8 & 19.7 & 19.6 & 17.1 & 20.3 & 19.4 & 20.1 \\
\hline Contacto contralateral & $\%$ & 49.6 & 50.3 & 50.0 & 48.7 & 54.7 & 48.4 & 52.8 \\
\hline Despegue & $\%$ & 59.0 & 68.0 & 69.6 & 67.7 & 71.6 & 65.2 & 71.7 \\
\hline Apoyo unipodal & $\%$ & 39.7 & 30.6 & 30.4 & 31.6 & 34.5 & 29.0 & 32.7 \\
\hline Apoyo bipodal & $\%$ & 19.3 & 37.4 & 39.2 & 36.1 & 37.2 & 36.1 & 39.0 \\
\hline Longitud de ciclo & $\mathrm{m}$ & 1.2 & 0.9 & 0.9 & 0.9 & 0.9 & 0.9 & 0.9 \\
\hline Longitud de paso & $\mathrm{m}$ & 0.6 & 0.5 & 0.4 & 0.5 & 0.4 & 0.5 & 0.4 \\
\hline
\end{tabular}

En la Tabla 3 se presentan los gráficos del análisis cinemático donde se disocia el movimiento de cada articulación en cada plano anatómico. Las filas muestran la cinemática angular de cada articulación (pelvis, cadera, rodilla y tobillo, comenzando desde arriba) y las columnas los planos de movimiento (plano frontal, sagital y transverso, comenzando desde la izquierda).

La pelvis en el plano frontal (Tabla 3a) muestra un rango de movimiento restringido pero simétrico, en tanto que en el plano sagital (Tabla 3b) se puede ver una leve tendencia hacia la retroversión con un patrón de doble resalto invertido. El plano transverso presenta una pelvis rotada (Tabla 3c), con la hemipelvis derecha retrasada a lo largo de todo el ciclo de marcha.

Las articulaciones de las caderas presentan una asimetría en el plano frontal con la derecha más aducida que la izquierda (Tabla 3d), en tanto que el rango de flexión-extensión está preservado, pero con una ligera disminución de la flexión al contacto inicial (Tabla 3e). En el plano transverso se registra un patrón asimétrico con la cadera derecha rotada internamente durante el apoyo con un marcado pico de rotación externa durante el balanceo, en tanto que la cadera izquierda se presenta levemente rotada hacia externo durante todo el ciclo de marcha (Tabla 3f).

La articulación de la rodilla izquierda presenta un adecuado patrón y rango de movimiento en el plano sagital con un leve aumento de la flexión al contacto inicial (Tabla 3h). La articulación de la rodilla derecha presenta una flexión sostenida de unos $9^{\circ}$ aproximadamente durante la mayor parte del apoyo, manteniendo este ángulo de flexión a lo largo del contacto inicial, respuesta a la carga, apoyo medio y terminal. En el plano transverso se puede observar una torsión tibial aumentada del lado derecho (Tabla 3i).

Finalmente, en relación con los ángulos de la articulación del tobillo y pie se puede observar un patrón de movimiento adecuado en el plano sagital, pero una leve disminución del rango de flexión plantar bilateral (Tabla 3j). En el plano transverso hay una leve asimetría de los ángulos de progresión de los pies (izquierdo hacia interno), con un importante pico de rotación externa del pie derecho en la fase de balanceo (Tabla 3k). 
Tabla 3 - Gráficas de cinemática. Verde: lado derecho. Rojo: Lado izquierdo.

a, b, c: Pelvis. d, e, f: Cadera. g, h, i: Rodilla. j, k: Tobillo/pie.

a, d, g: plano frontal. b, e, h, j: plano sagital. c, f, i, k: plano transverso
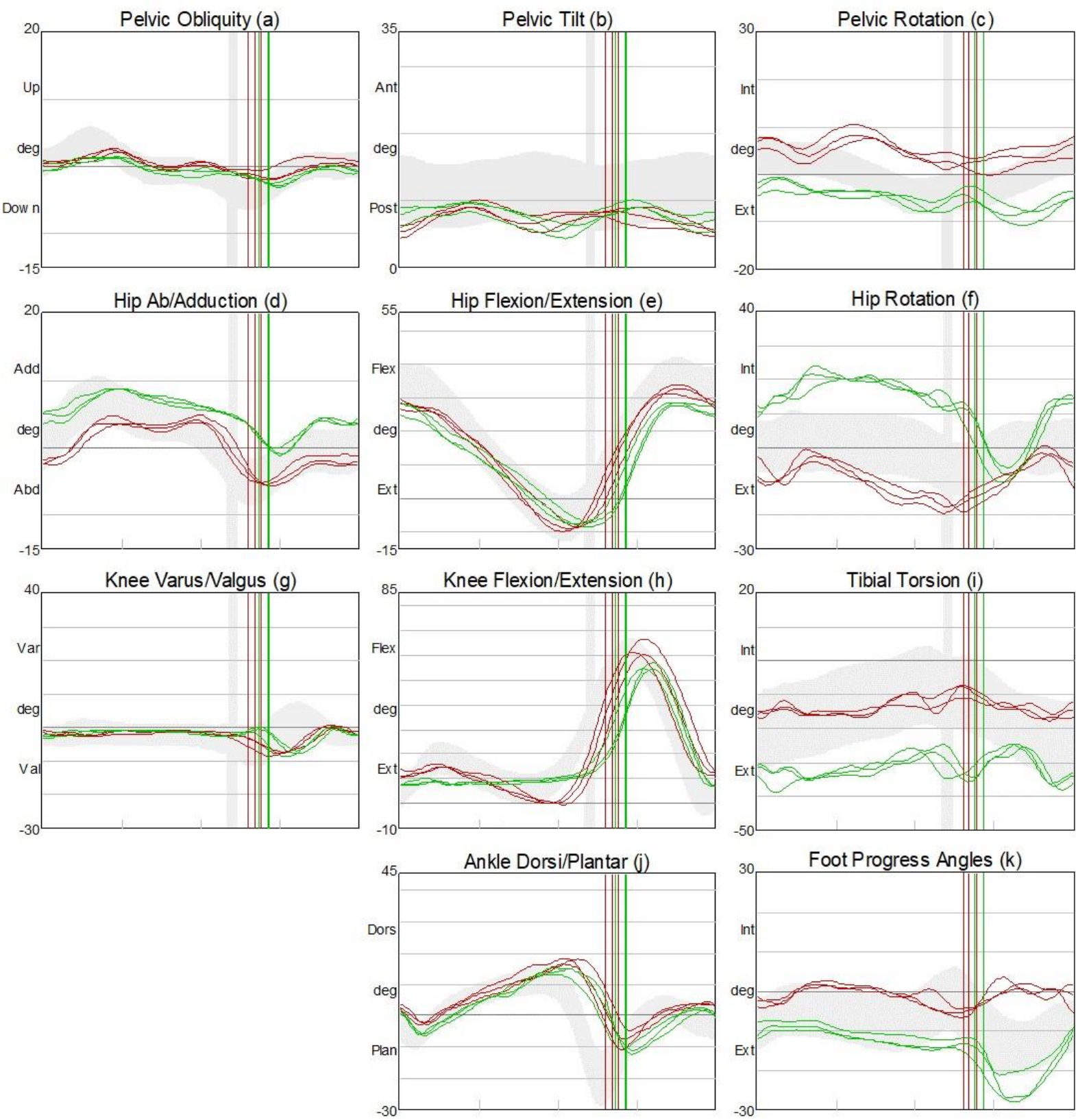


\section{Discusión}

Ante la escasez de publicaciones sobre el análisis de la marcha en este tipo de pacientes y con el tratamiento quirúrgico realizado, si bien se encontraron con tratamiento ortésico (2627), se podrían hacer algunas conjeturas acerca de las posibles causas de las diferentes adaptaciones que se observaron, para que la paciente logre una marcha funcional.

La longitud de ciclo y velocidad reducidos durante la marcha (Tabla 2) podría deberse a la esperable falta de estabilidad general, que se relaciona con la debilidad (Tabla 1) de los grupos musculares necesarios para una marcha estable.

El hecho constatado de que los pasos con la extremidad inferior derecha sean más cortos (Tabla 2) podría deberse a las diferencias existentes en la valoración de la fuerza muscular de la musculatura flexora plantar de tobillo de la pierna izquierda $(2 / 5)$ con respecto a la derecha (3/5; Tabla 1). El paciente reacciona a la debilidad de la musculatura flexora plantar reduciendo su demanda, utilizando como estrategia una limitación de la progresión mediante la toma de pasos más cortos con la extremidad contralateral (3).

La retroversión pélvica podría deberse a la debilidad de la musculatura extensora del raquis lumbar (constatada durante la valoración física, 3/5) debido a las secuelas de la poliomielitis (3). Esta retroversión pélvica favorece la activación de músculo Iliaco durante la fase de oscilación y el aporte de momento flexor por parte de las estructuras pasivas periarticulares de la cadera en forma de resistencia de los ligamentos a la extensión de dicha articulación (28). Además, la retroversión pélvica posiciona al músculo Tensor de la Fascia Lata (TFL) en una relación de longitud-tensión tal que se encuentre dentro del rango de tensión máxima activa y en una buena posición de tensión pasiva, de forma que se favorezca al máximo la función de este músculo sobre la extremidad inferior en las demandas de abducción, rotación interna y flexión de cadera. La función de este músculo es extremadamente importante durante la fase de oscilación final para la estrategia de marcha que se observa en este caso (14). Cuando la retroversión se produce durante la segunda mitad de la fase de balanceo puede deberse a un acortamiento de la musculatura isquiosural, teoría que se rechazó dado que no se objetivó dicho acortamiento en la valoración del ángulo poplíteo unilateral ni en el bilateral (3; Tabla 1).

Además, una mayor tensión relativa del Glúteo Mayor (GM) respecto a los flexores de cadera podría estar contribuyendo en la retroversión pélvica aumentada. Esto podría deberse a la doble tarea que realiza este músculo para generar extensión indirecta de la articulación de la rodilla traccionando del fémur hacia posterior a través de su inserción proximal en dicho hueso, y extensión directa de la misma a través de su inserción en la cintilla iliotibial (3). Estas acciones del GM son necesarias desde la fase oscilación final hasta la mitad del apoyo medio.

Los pacientes que contrajeron polio durante la primera infancia pueden adolecer de caída pélvica (Pelvic Drop) y por consiguiente presentar una restricción del movimiento pélvico en el plano frontal (3). Esto puede deberse a que presenten una extremidad más corta que la otra, que genere un pre posicionamiento pélvico y dado que la paciente no presenta dicha dismetría (Tabla 1), cobra fuerza la posibilidad de que haya desarrollado una retracción de la cintilla iliotibial derecha como adaptación para brindar estabilidad pasiva a la pelvis y a toda la extremidad inferior (3).

La marcada rotación interna (RI) de la articulación de la cadera derecha durante la oscilación media y final observada podría deberse a diferentes factores. Por un lado, podría servir para favorecer la activación del TFL de forma que su acción como abductor de cadera sea transformada en flexión de la misma gracias a la rotación del eje de flexión-extensión y a su vez estabilizando la articulación de la rodilla en la máxima extensión de la que dispone la 
paciente $\left(9^{\circ}\right.$ de flexión; Tabla 1$)$. El músculo TFL a través de su inserción en la tibia por la cintilla iliotibial actúa como extensor de rodilla entre $0-30^{\circ}$ de flexión y como flexor de la misma desde $30^{\circ}$ de flexión en adelante (29). Recordando que durante las fases mencionadas anteriormente en circunstancias normales, debería ser el cuádriceps el encargado de proporcionar esa función doble de flexor de cadera (colaborador en la flexión de cadera) a través del recto anterior y que los vastos deberían activarse durante la fase de oscilación final para asegurar la extensión de la articulación de la rodilla, así como para preparar a esta para las altas demandas funcionales asociadas con la brusca caída del peso corporal durante la aceptación del peso/respuesta a la carga (3). La paciente presenta un valor de fuerza del cuádriceps de la extremidad inferior derecha menor que la antigravitatoria (Tabla 1) y se ve obligada a desarrollar estrategias alternativas para tener una marcha funcional.

Por otro lado, la rotación interna de la articulación de la cadera predispone los cóndilos femorales durante el contacto inicial de tal forma que consigue rotar el eje natural de flexiónextensión de la articulación de la rodilla evitando que la elevada energía que se produce en la fase inmediatamente posterior de la respuesta a la carga no consiga claudicar en flexión de rodilla (30). Así, absorbiéndolo en forma de bostezo/varo forzado del compartimento lateral de la prótesis, dada la debilidad del músculo cuádriceps que lo incapacita para ralentizar de forma excéntrica la rápida flexión de rodilla que se da como uno de los varios mecanismos de absorción de impacto al contactar la extremidad inferior con el suelo de forma abrupta.

Por último, la rotación interna de la articulación de la cadera durante todo el apoyo podría tratar de compensar la notable torsión tibial externa que presenta la paciente (Tabla 1) para favorecer la actividad del tríceps sural debilitado (3/5) en un intento por retener el avance de la tibia con respecto al avance del fémur.

A partir de la oscilación final y hasta la mitad del apoyo medio, es la actividad del GM la que proporciona la fuerza de extensión de la articulación de la cadera y la estabilidad en extensión de la articulación de la rodilla mediante la inserción de la cintilla iliotibial sobre el borde anterolateral de la tibia, como se mencionó anteriormente.

Durante el balanceo inicial se produce un importante movimiento de rotación externa de la articulación de la cadera derecha. Esta rotación podría deberse a la liberación súbita de la extremidad inferior que se encontraba en marcada rotación interna durante el apoyo medio y terminal, y que al finalizar la restricción del contacto con el suelo produce una liberación de energía elástica. Otra hipótesis para la mencionada rotación externa es que la flexión de la articulación de la cadera y de la articulación de la rodilla necesarias en el balanceo inicial sean producidas principalmente por el músculo sartorio, que además de ser flexor de la articulación de la cadera y de la articulación de la rodilla es rotador externo de cadera.

La actividad del psoas como flexor de la articulación de la cadera en la fase de oscilación inicial podría generar una rotación externa de la misma, dado que esta es su función secundaria. La actividad de este músculo podría verse favorecida por el alejamiento de su origen en la región lumbar dictada por la posición de retroversión pélvica anteriormente mencionada (14).

La diferencia en la abducción y aducción de ambas articulaciones de las caderas podría deberse a la rotación de la pelvis en el plano transversal que transforma parte de la flexión y extensión de cadera en abducción y aducción de esta.

La posición cuasi invariable de flexión de la articulación de la rodilla de $9{ }^{\circ}$ durante el contacto inicial, la respuesta a la carga, el apoyo medio y el terminal, se corresponde con la valoración articular del rango de movimiento pasivo de rodilla, cuyo límite hacia la extensión 
es de $-9^{\circ}$. Se puede observar, además, que la vista sagital de la radiografía postquirúrgica (Figura 2) muestra una prótesis en extensión completa, mientras que la posición de la articulación no se corresponde con la misma, y sí con un flexo de rodilla de $9^{\circ}$. Esto podría deberse al reto técnico que supone una ATR en pacientes con secuelas de poliomielitis en los que generalmente se presentan, como se mencionó anteriormente, deformidades articulares y metafisarias, aumentada rotación tibial externa, valgos excesivo, pobre calidad del hueso, genu-recurvatum y laxitud ligamentosa, características que pueden alterar los procesos intrínsecos de la cirugía, como la realización de los canales para los vástagos tibial y femoral (1-2). También así la colocación adecuada de dichos vástagos y el fraguado del cemento, que al tratarse habitualmente de huesos osteopénicos, puede generar con facilidad alteraciones no deseadas en la alineación del el elemento protésico (8).

Figura 2 - Radiografía postquirúrgica de artroplastia total de rodilla derecha

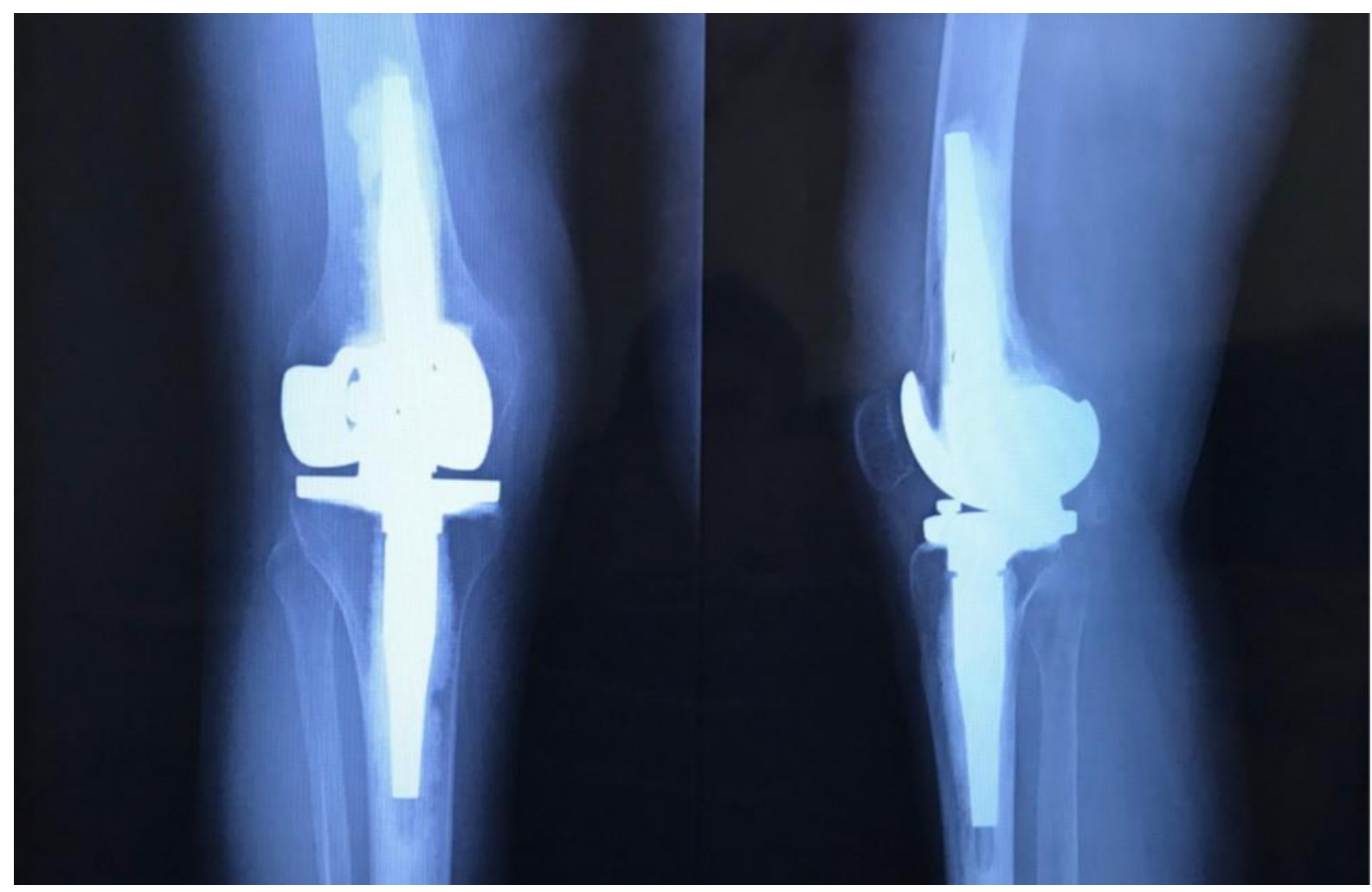

Entre las limitaciones de este estudio destaca el hecho de que en el mismo se analiza únicamente los resultados de la cinemática de la marcha, ya que no se cuenta con otro equipamiento como plataformas de fuerza o electromiografía, que ocasionalmente están presentes en este tipo de estudios. El análisis de la cinética (momentos de fuerza y potencia de las articulaciones), o el registro de la actividad eléctrica muscular, podría arrojar otro tipo de información para un análisis aún más profundo y exhaustivo (31-32). Se carece de caracterizaciones de la marcha previo a la intervención de ATR, de forma que no se puede confirmar si las variaciones con respecto a la marcha normal observadas en este estudio y la estrategia de marcha descrita anteriormente era utilizada por el paciente previo a la intervención quirúrgica, o si la adquirió en la etapa de rehabilitación y readaptación. La debilidad y la activación retrasada del músculo cuádriceps han sido identificadas como las mayores causas de limitaciones en la funcionalidad tras ATR (33-34). 
Se concluye que ya que la debilidad del músculo cuádriceps (2/5) es una secuela de la poliomielitis (1), la paciente utilizaba una estrategia de empuje extensor (extensor thrust, 3), en la que el GM genera una extensión indirecta de la articulación de la rodilla a través de su inserción en el fémur y a su vez bloquea la misma en extensión completa o hiperextensión (habitual en pacientes con secuelas de poliomielitis), por medio de la inserción distal de la cintilla iliotibial en la tibia y que evita la claudicación de esta articulación en la fase de respuesta a la carga.

Los autores de este trabajo comparten la idea de que se produzca el empuje extensor, pero añaden y resaltan la posible importancia del músculo TFL en las fases de la marcha en las que se requiere una extensión o mantenimiento de la rodilla en los $9^{\circ}$ de flexión. Como la articulación de la cadera se encuentra en flexión y el GM no es capaz de producir su triple función como extensor de la misma, extensor indirecto de la articulación de la rodilla y de mantenimiento de esta última bloqueada en extensión, es el TFL el encargado de preparar la extremidad durante la oscilación media y final de forma que el contacto inicial se produzca en la máxima extensión de la que dispone la articulación de la rodilla derecha. Es ahí donde el GM se encuentra en disposición de acometer su tarea.

Con la información recabada, tanto en la valoración física como con la caracterización de la cinemática de la marcha, se plantea que ante el déficit de fuerza del cuádriceps de la extremidad inferior derecha (irreversible debido a las secuelas del síndrome postpoliomielítico), se podría pensar en la potenciación de los músculos utilizados para suplir las funciones abolidas, como los flexores y rotadores de la articulación de la cadera, el TFL y el sartorio, y extensores de la misma articulación así como la musculatura estabilizadora lumbopélvica, el GM y medio. A su vez, el TFL y el GM colaboran a nivel distal con el mantenimiento de la extensión de la articulación de la rodilla durante la respuesta a la carga, por lo que se considera importante enfocar la planificación terapéutica hacia los mencionados grupos musculares.

\section{Referencias}

1. Patterson BM, Insall JN. Surgical management of gonarthrosis patients with poliomyelitis. J Arthroplasty. 1992; 7(Suppl.): 419-426.

2. Giori NJ, Lewallen DG. Total knee arthroplasty in limbs affected by poliomyelitis. J Bone Joint Surg. 2002; 84(7): 1157-1161.

3. Perry J, Burnfield JM. Gait Analysis: Normal and Pathological Function. $2^{\mathrm{a}}$ ed. New Jersey: Slack Incorporated; 2010.

4. Organización Mundial de la Salud. Notificados a la OMS el 1 de marzo de 2019. Poliomielitis [Internet]. Ginebra: OMS; 2020 [consultado 24 oct 2020]. Disponible en: https://www.who.int/es/news-room/fact-sheets/detail/poliomielitis.

5. Rahman J, Hanna SA, Kayani B, Miles J, Pollock RC, Skinner JA, et al. Custom rotating hinge total knee arthroplasty in patients with poliomyelitis affected limbs. Int Orthop. 2015; 39(5): 833-838.

6. Jordan L, Kligman M, Sculco TP. Total knee arthroplasty in patients with poliomyelitis. J Arthroplasty 2007; 22(4): 543-548.

7. Tigani D, Fosco M, Amendola L, Boriani L. Total knee arthroplasty in patients with poliomyelitis. Knee. 2009; 16(6): 501-506.

8. Prasad A, Donovan R, Ramachandran M, Dawson-Bowling S, Milington S, Bhumbra Rej, et al. Outcome of total knee arthroplasty in patients with poliomyelitis: A systematic review. EFORT Open Rev. 2018; 3(6): 358-362.

9. Dauwe J, Vandenneucker H. Indications for primary rotating-hinge total knee arthroplasty. Is there consensus? Acta Orthop Belg. 2018; 84(3): 245-250. 
10. Partezani C, Nogueira P, Maftoum C, Gomes R, Kawamura M, Luis G. Knee arthroplasty with rotating -hinge implant: an option for complex primary cases and revisions. Rev Bras Ortop. 2018: 53(2); 151-157.

11. Yang JH, Yoon JR, Oh CH, Kim TS. Primary total knee arthroplasty using rotatinghinge prosthesis in severely affected knees. Knee Surg Sports Traumatol Arthrosc. 2012: 20(3); 517-523.

12. Kirschberg J, Goralski S, Layher F, Sander K, Matziolis G. Normalized gait analysis parameters are closely related to patient-reported outcome measures after total knee arthroplasty. Arch Orthop Trauma Surg. 2018; 138(5): 711-717.

13. Dominguez F, Igual C, Silvestre A, Roig S, Blasco JM. Effects of balance and proprioceptive training on total hip and Knee replacement rehabilitation: A systematic review and meta-analysis. Gait Posture. 2018; 62: 68-74.

14. Kendall FP, McCreary EK. Kendall's músculos: Pruebas funcionales, postura y dolor. 5a. ed. Madrid: Marbán; 2007.

15. Taboadela C. Goniometría: una herramienta para la evaluación de las discapacidades laborales. $1^{\text {a }}$ ed. Buenos Aires: Asociart ART; 2007.

16. Davis RB, Ounpuu S, Tyburski D GJ. A gait analysis data collection and reduction technique. Hum Mov Sci. 1991; 10: 575-587.

17. Kadaba MP, Ramakrishnan HK . Measurement of Lower Extremity Kinematics During Level Walking. J Orthop Res. 1990; 8: 383-892.

18. McGinley JL, Baker R, Wolfe R, Morris ME. The reliability of three-dimensional kinematic gait measurements: A systematic review. Gait Posture. 2009; 29(3): 360-369.

19. Baker R. Gait analysis methods in rehabilitation. J Neuroeng Rehabil. 2006; 3: 1-10.

20. Cappozzo A, Catani F, Della Croce AL. Position and orientation in space of bones during movement: anatomical frame definition and determination. Clin Biomech. 1995; 10(4): 171-178.

21. Collins TD, Ghoussayni SN, Ewins DJ, Kent JA. A six degrees-of-freedom marker set for gait analysis: Repeatability and comparison with a modified Helen Hayes set. Gait Posture. 2009; 30(2): 173-180.

22. Schwartz MH, Rozumalski A. A new method for estimating joint parameters from motion data. J Biomech. 2005 Jan; 38(1): 107-116.

23. Ehrig RM, Taylor WR, Duda GN, Heller MO. A survey of formal methods for determining the centre of rotation of ball joints. J Biomech [Internet]. 2006; 39(15): 2798-2809. Disponible en: http://www.ncbi.nlm.nih.gov/pubmed/16293257

24. Camomilla V, Cereatti A, Vannozzi G, Cappozzo A. An optimized protocol for hip joint centre determination using the functional method. J Biomech. 2006; 39(6): 10961106.

25. Ehrig RM, Taylor WR, Duda GN, Heller MO. A survey of formal methods for determining functional joint axes. J Biomech [Internet]. 2007; 40(10): 2150-2157. Disponible en: http://www.ncbi.nlm.nih.gov/pubmed/17169365

26. Portnoy S, Schwartz I. Gait characteristics of post-poliomyelitis patients: Standardization of quantitative data reporting. Ann Phys Rehabil Med. 2013; 56(7-8): 527-541.

27. Brogardh C, Flansbjer VB, Lexell J. Determinants of falls and fear of falling in ambulatory persons with late effects of polio. PM R. 2017; 9(5): 455-463.

28. Simonsen E, Cappelen K, Skorini R, Larsen P, Alkjær T, Dyhre-Poulsen P. Explanations pertaining to the hip joint flexor moment during the stance phase of human walking. J Appl Biomech. 2012; 28(5): 542-550. 
29. Trammell AP, Nahian A, Pilson H. Anatomy, Bony Pelvis and Lower Limb, Tensor Fasciae Latae Muscle [citado May 7 2020]. En: StatPearls [Internet]. Disponible en: https://www.ncbi.nlm.nih.gov/books/NBK499870/

30. Kapandji A. Fisiología articular: Miembro inferior. Tomo 2. $1^{a}$ ed. Madrid: Panamericana; 2012.

31. Elkarif V, Kandel L, Rand D, Schwartz I, Greenberg A, Portnoy S. Muscle activity while ambulating on stairs and slopes: A comparison between individuals scheduled and not scheduled for knee arthroplasty and healthy controls. Musculoskeletal Science and Practice. 2021; 52: 102346.

32. Bianchi N, Facchini A, Mondanelli N, Sacchetti F, Ghezzi R, Gesi M, Capanna R, Giannotti S. Medial pivot vs posterior stabilized total knee arthroplasty designs: a gait analysis study. Med Glas (Zenica). 2021 Feb 1; 18(1): 252-259.

33. Yoshida Y, Mizner RL, Snyder-Mackler L. Association between long-term quadriceps weakness and early walking muscle co-contraction after total knee arthroplasty. Knee. 2013; 20(6): 426-431.

34. Marmon AR, Snyder- Mackler L. Activation deficits do not limit quadriceps strength training gains in patients after total knee arthroplasty. Int J Sports Phys Ther. 2014; 9(3): 329-337.

Cómo citar: López D, Peña N, Benítez E, Samudio S, Vecino P, Benvenutto E. Análisis biomecánico de la marcha en paciente con secuelas de poliomielitis y artroplastia de rodilla. Enfermería: Cuidados Humanizados. 2021; 10(1): 75-88. Doi: https://doi.org/10.22235/ech.v10i1.2359

Contribución de los autores: a) Concepción y diseño del trabajo, b) Adquisición de datos, c) Análisis e interpretación de datos, d) Redacción del manuscrito, e) Revisión crítica del manuscrito.

DL ha contribuido en a, b, c, d, e; NP ha contribuido en a, b, c, d, e.; EB ha contribuido en a, d, e; SS ha contribuido en a, b, c; PV ha contribuido en a, b, c; EB ha contribuido en a, b, c.

Editora científica responsable: Dra. Natalie Figueredo. 